OPEN ACCESS

Edited by:

Jiri Jiracek,

Institute of Organic Chemistry and Biochemistry (ASCR), Czechia

Reviewed by:

Lenka Maletinska,

Institute of Organic Chemistry and

Biochemistry (ASCR), Czechia

Åke Sjöholm

Gävle Hospital, Sweden

${ }^{*}$ Correspondence:

Jian-xun Wang

wangjx@qdu.edu.cn

Xue-juan Zhang

dzhangxue@126.com

${ }^{\dagger}$ These authors have contributed equally to this work

Specialty section:

This article was submitted to

Diabetes,

a section of the journal

Frontiers in Endocrinology

Received: 11 January 2019

Accepted: 12 March 2019

Published: 05 April 2019

Citation:

Ding W, Chang W, Guo X, Liu Y, Xiao

$D$, Ding $D$, Wang $J$ and Zhang $X$ (2019) Exenatide Protects Against

Cardiac Dysfunction by Attenuating

Oxidative Stress in the Diabetic Mouse Heart. Front. Endocrinol. 10:202 doi: 10.3389/fendo.2019.00202

\section{Exenatide Protects Against Cardiac Dysfunction by Attenuating Oxidative Stress in the Diabetic Mouse Heart}

\author{
Wei Ding ${ }^{1 \dagger}$, Wen-guang Chang ${ }^{2 \dagger}$, Xiao-ci Guo ${ }^{1}$, Ying Liu ${ }^{2}$, Dan-dan Xiao ${ }^{2}$, Dan Ding ${ }^{2}$, \\ Jian-xun Wang ${ }^{2,3 *}$ and Xue-juan Zhang ${ }^{1 *}$ \\ ${ }^{1}$ Department of General Medicine, The Affiliated Hospital, Qingdao University, Qingdao, China, ${ }^{2}$ Center for Regenerative \\ Medicine, Institute for Translational Medicine, Qingdao University, Qingdao, China, ${ }^{3}$ School of Basic Medical Sciences, \\ Qingdao University, Qingdao, China
}

Cardiovascular disease is the major cause of death in patients with diabetes. Current treatment strategies for diabetes rely on lifestyle changes and glucose control to prevent angiopathy and organ failure. Exenatide, a glucagon-like peptide-1 (GLP-1) receptor agonist, is used as an add-on therapy to insulin treatment. Exenatide also has multiple beneficial effects in addition to its hypoglycemic effects, such as preventing hepatic steatosis and protecting against cardiac injury from doxorubicin-induced cardiotoxicity or ischemic reperfusion. However, the mechanisms underlying the cardioprotective effects of exenatide in diabetes have not been fully clarified. To address this issue, we investigated the cardioprotective effects of exenatide in type 1 and type 2 diabetic mice. We found that exenatide simultaneously attenuated reactive oxidative species (ROS) production through increases in the antioxidant enzymes manganese dependent superoxide dismutase (MnSOD) and catalase. Moreover, exenatide decreased tumor protein P53 (p53) expression and prevented cell apoptosis in H9c2 cells. The presence of the catalase inhibitor 3-AT attenuated the effects of exenatide. Overall, the results strongly indicate that exenatide treatment may be protective against the development of diabetic cardiomyopathy.

Keywords: exenatide, diabetes, ROS, apoptosis, cardiac function

\section{INTRODUCTION}

Diabetes mellitus is a metabolic disorder characterized by hyperglycemia. Cardiovascular disease is the major cause of death in diabetic patients. Patients with diabetes are at least 10 times more likely to suffer from heart dysfunction than their non-diabetic counterparts (1-3). Current treatment strategies for diabetes rely on lifestyle changes and glucose control to prevent angiopathy and organ failure. Although many hypoglycemic agents have been extensively investigated in recent years, there is still a need for effective therapeutic strategies for better results.

GLP-1 is an insulinotropic hormone released from intestinal L cells in response to nutrient ingestion, and it modulates glucose metabolism by affecting pancreatic islet secretions. However, it can be rapidly inactivated by the enzyme dipeptidyl peptidase IV in the circulation. Exenatide is a kind of GLP-1 analog that can evade rapid clearance by dipeptidyl peptidase IV; thus, it has a long half-life when injected subcutaneously. Exenatide was approved for type 2 diabetic treatment in 2005. Previous studies have shown that in addition to the hypoglycemic effect, exenatide reduced weight gain and alleviated hepatic steatosis in diabetic mice $(4,5)$. In 
addition, several studies demonstrated that exenatide has beneficial effects on cardiac function. In a clinical trial, a study was conducted on 23 patients with uncomplicated type 2 diabetes. Administration of exenatide (10 $\mu \mathrm{g}$, bid) improved cardiac function and reduced arterial stiffness (6). Another clinical study also showed that exenatide twice daily as an add-on therapy was associated with significant, sustained improvement in several cardiovascular risk markers in patients with type 2 diabetes vs. glimepiride treatment (7). In addition, adjunctive exenatide administered with primary percutaneous coronary intervention was shown to reduce infarct size and improve subclinical left ventricular function in patients with ST segment elevation myocardial infarction (8). Consistent with these findings, in animal studies, streptozotocin-induced diabetic rats that underwent a 12-week treatment with exenatide exhibited significantly improved cardiac function, glucose uptake, and microvascular barrier function (9). Exenatide pretreatment improved cardiac function and reduced the infarct area in rat hearts after ischemia reperfusion (10). Additionally, exenatide protects cardiomyocytes against doxorubicin-induced cardiotoxicity (11).

The pathological mechanisms underlying diabetic cardiomyopathy, including inflammation, mitochondria dysfunction, lipotoxicity, and oxidative stress, are complex and multifactorial. The molecular mechanisms underlying the cardioprotective effects of exenatide remain unclear. It has been reported that exenatide prevents cardiomyocyte apoptosis by enhancing autophagy and reducing lipotoxic ceramide accumulation $(12,13)$, as well as preventing mitochondrial dysfunction and endoplasmic reticulum stress (14, 15). Oxidative stress and accelerated reactive oxidative species (ROS) production induced by high glucose are known to play key roles in the progression of diabetic cardiovascular disease and cardiomyocyte apoptosis. We hypothesize that exenatide could exert its cardioprotective effects by preventing ROS production (16-18). Thus, this study was designed to determine the protective effects of exenatide on cardiac functions in mouse models of both type 1 and type 2 diabetes and to explore the underlying molecular mechanism. In vivo and in vitro studies were performed to assess cardiac function, glucose tolerance, antioxidative stress capacity and cell apoptosis. The effects of exenatide on ROS production and apoptosis were evaluated in $\mathrm{H} 9 \mathrm{c} 2$ cardiomyoblasts. Furthermore, the underlying mechanism of exenatide-induced protective effects was investigated. Our study provides a new mechanism underlying the cardioprotective actions of exenatide.

\section{MATERIALS AND METHODS}

\section{Animal Model}

Six-week-old male C57BL/6J mice were purchased from Beijing Vital River Laboratory Animal Technology. The mice were

Abbreviations: ROS, reactive oxidative species; GLP-1, glucagon-like peptide1; IPGTT, intraperitoneal glucose tolerance test; MnSOD, manganese-dependent superoxide dismutase; MAPK, mitogen-activated protein kinases; P53, tumor protein P53. housed in an environmentally controlled breeding room (temperature: $20 \pm 2{ }^{\circ} \mathrm{C}$, humidity: $60 \pm 5 \%, 12 / 12$ light-dark cycle). All mice had free access to tap water. All procedures were approved by the Ethics Committee for the Use of Experimental Animals of Qingdao University.

\section{Type 2 Diabetic Mice}

For the high-fat diet-induced type 2 diabetic mouse study, mice were randomly distributed into two initial groups fed a control diet $(10 \% \mathrm{Kcal}$ fat, $70 \% \mathrm{Kcal}$ carbohydrate, and $23 \% \mathrm{Kcal}$ protein with a total caloric value of $3.85 \mathrm{Kcal} / \mathrm{gm}$. Research diet D12450B, CON group, $n=8$ ) or a high-fat diet (consisting of $45 \% \mathrm{Kcal}$ fat, $35 \% \mathrm{Kcal}$ carbohydrate, and $20 \% \mathrm{Kcal}$ protein with a total caloric value of $4.73 \mathrm{Kcal} / \mathrm{gm}$. Research diet D12451 HFD group, $n=16$ ). After a 20-week dietary intervention, the HFD group was then randomly subdivided to receive either exenatide $(24 \mathrm{nmol} / \mathrm{kg} / \mathrm{d}$, BYETTA; T2DM+EXE group, $n=8$ ) or normal saline as a control (T2DM group, $n=8$ ) by intraperitoneal injection during the light cycle while continuing the HFD feeding for 4 weeks.

\section{Type 1 Diabetic Mice}

Type 1 diabetes was induced by treating the mice with a high dose of streptozotocin (STZ) (Sigma-Aldrich, Shanghai, China) as previously described (19). Briefly, the mice received an intraperitoneal injection of STZ at a dose of $120 \mathrm{mg} / \mathrm{kg}$ (dissolved in $0.1 \mathrm{~mol} / \mathrm{L}$ citrate buffer, $\mathrm{pH} 4.5$ ). To observe the diabetic status of the mice, the non-fasting glucose level was monitored from tail blood samples by a glucose meter (Roche, ACCUCHEK, Performa glucometer). Diabetes onset was diagnosed when the blood glucose level was $>16.7 \mathrm{mmol}(300 \mathrm{mg} / \mathrm{dl})$ on 2 consecutive tests. Then, the type 1 diabetic mice were randomly separated into two groups that were both fed a control diet. The mice then received either exenatide $(24 \mathrm{nmol} / \mathrm{kg} / \mathrm{d}$, BYETTA; T1DM+EXE group, $n=8$ ) or normal saline as a control (T1DM group, $n=8$ ) by intraperitoneal injection during the light cycle while continuing feeding with the chow diet for 4 weeks.

\section{Intraperitoneal Glucose Tolerance Test (IPGTT)}

After 24 weeks, body weights were measured in all groups. However, the glucose tolerance test ( $n=8$ in each group) was measured only in type 2 diabetic mice. Briefly, the IPGTT was conducted after an overnight fast $(12-16 \mathrm{~h})$. Mice were injected with $40 \%$ glucose ( $2 \mathrm{~g} / \mathrm{kg}$ body weight, i.p.). Blood glucose was measured from the tail tip using a glucose meter (Roche, ACCUCHEK, Performa glucometer) at 0, 30, 60, 90, and $120 \mathrm{~min}$.

\section{HOMA-IR Measurement}

Plasma insulin ( $n=8$ in each group) was measured using a commercial ELISA kit (Beyotime, China). Homeostasis model assessment (HOMA-IR) was calculated by fasting plasma glucose $(\mathrm{FPG}, \mathrm{mmol} / \mathrm{L}) \times$ fasting plasma insulin $(\mathrm{FINS}, \mathrm{mIU} / \mathrm{L}) / 22.5$.

\section{Echocardiography}

After 24 weeks, the mice in each group were anesthetized using $0.3 \%$ pentobarbital sodium (intraperitoneal injection). Transthoracic two-dimensional M-mode echocardiography and 
pulsed wave Doppler spectral tracings were obtained using a Vevo 2100 Imaging System (VisualSonics Inc., Canada) with a $30-\mathrm{MHz}$ transducer for each mouse. The percentages of ejection fraction (EF\%), left ventricular end-systolic diameter (LVSD), and left ventricular end-diastolic diameter (LVDD) were measured using M-mode tracings. The percentage of fractional shortening (FS\%) was calculated according to the following formula [(LVDD-LVSD)/LVDD] $\times 100 \%$. The ratio of early ventricular filling peak velocity ( $\mathrm{E}$ wave) and late filling velocity (A wave) (E/A) were measured by pulsed wave Doppler spectral tracings to determine the diastolic function.

\section{Cell Culture}

H9c2 cardiomyoblasts were purchased from Cobioer (Nanjing, China, ATCC origin). H9c2 cells were maintained in highglucose Dulbecco's modified Eagle's medium (DMEM, glucose $25 \mathrm{mM}$ ) with $10 \%$ fetal bovine serum (FBS), 2\% L-glutamine, $10 \%$ sodium bicarbonate, $10 \%$ sodium pyruvate, 5\% HEPES, $1 \%$ penicillin/streptomycin, and $1 \%$ gentamycin in an incubator $\left(37^{\circ} \mathrm{C}, 5 \% \mathrm{CO} 2\right)$. For high-glucose treatment, H9c2 cells were incubated for $24 \mathrm{~h}$ in high-glucose medium $(40 \mathrm{mM})$ with or without exenatide present ( $25 \mathrm{nM}, 50 \mathrm{nM})$.

\section{TUNEL Assay}

The terminal deoxynucleotidyl transferase-mediated deoxyuridine triphosphate nick-end labeling (TUNEL) assay was carried out according to the manufacturer's protocol with a one-step TUNEL apoptosis assay kit (Beyotime, Shanghai, China). The results were scored semiquantitatively by averaging the numbers of TUNEL-positive cells per low-power field for five fields in each experiment.

\section{ROS Determination}

$\mathrm{H} 9 \mathrm{c} 2$ cells in the presence of control medium or high-glucose medium $(40 \mathrm{mM})$ were treated with or without exenatide $(25$ or $50 \mathrm{nM}$ ) in the dark with $10 \mu \mathrm{M}$ DCFH-DA (Beyotime Biotechnology, Shanghai, China) for $20 \mathrm{~min}$. To observe DCF fluorescence during illumination, cells were mounted on a microscope slide and illuminated and visualized with a $20 \times$ objective on a Nikon-TI SR microscope. The number of positive cells was counted in at least three random pictures in each group.

To further quantify ROS production, H9c2 cells were seeded in 96-well plates in the presence of high glucose and treated with or without exenatide ( 25 or $50 \mathrm{nM}$ ). After $10 \mu \mathrm{M}$ DCFH-DA labeling, the fluorescence intensity of the cells was recorded by a fluorescence microplate reader at $488 / 525 \mathrm{~nm}$.

\section{Western Blotting Analysis}

Hearts from each group of animals were homogenized, and protein samples were prepared with ice-cold RIPA lysis buffer. Protein concentrations were measured using the Bradford assay (Bio-Rad protein assay kit). For western blotting detection of p53, catalase, MnSOD, and glyceraldehyde 3phosphate dehydrogenase (GAPDH) protein expression, $80 \mu \mathrm{g}$ of protein was used. All proteins were separated by $12 \%$ SDS polyacrylamide gel and electrotransferred onto polyvinylidene fluoride membranes (PVDF). Membranes were blocked with 5\% $(\mathrm{w} / \mathrm{v})$ skim milk for $2 \mathrm{~h}$ at room temperature and then incubated with primary antibodies with gentle agitation overnight at $4^{\circ} \mathrm{C}$. The membranes were washed 3 times for $10 \mathrm{~min}$ each with $15 \mathrm{ml}$ of TBST (10 mM Tris- $\mathrm{HCl}, 150 \mathrm{mM} \mathrm{NaCl}$ and $0.1 \%(\mathrm{v} / \mathrm{v})$ Tween-20 and then incubated with secondary antibody at room temperature for $2 \mathrm{~h}$.

For in vitro western blotting experiments, upon completion of the experiments, H9c2 cells were harvested using ice-cold RIPA lysis buffer. Protein concentrations were measured using the Bradford assay (Bio-Rad protein assay kit). For western blotting detection of P53, catalase and MnSOD protein expression, the method used is the same as that described above.

Proteins for in vivo and in vitro experiments were visualized with enhanced chemiluminescence solution and images generated with a GENE Imaging system. The images were quantified using Image Analysis Software (Quantity One). Primary antibodies for p53 (catalog number, 60283-2Ig), catalase (catalog number, 21260-1-AP), and MnSOD (catalog number, 66474-1-Ig) were purchased from Proteintech (Proteintech Group, Inc., USA). GAPDH and HRP-conjugated secondary antibodies were purchased from Yesen Biotechnology (Shanghai, China).

\section{Statistical Analysis}

Data are presented as the mean \pm SEM. An unpaired two-tailed Student's $t$-test was used to determine significant differences between groups. For comparison of more than two groups, oneway analysis of variance (ANOVA) followed by Tukey's post hoc test was performed. Adjusted two-sided $P$-values were calculated, and $P<0.05$ was considered indicative of statistical significance.

\section{RESULTS}

\section{Exenatide Treatment Reduces Body Weight and Attenuates Glucose Intolerance in Type 2 Diabetic Mice}

The mean body weights of the CON, T2DM, T2DM+EXE and T1DM, T1DM+EXE animals were measured. The body weight of T2DM mice was increased compared to that of the CON mice, and treatment of T2DM animals with exenatide (24 $\mathrm{nmol} / \mathrm{kg} / \mathrm{d}$ ) decreased body weight significantly. T1DM mice had significantly reduced body weight compared to the CON mice, and treatment of T1DM animals with exenatide $(24 \mathrm{nmol} / \mathrm{kg} / \mathrm{d})$ slightly increased body weight, although the difference was not significant (Figure 1A). In addition, fasting blood glucose (FBG) levels in both T1DM and T2DM groups was increased compared to that in the CON group, and exenatide-treated mice showed decreased FBG levels compared to model animals (Figure 1B). Moreover, the intraperitoneal glucose tolerance test and Homeostasis model assessment (HOMA-IR) levels showed that exenatide improved glucose tolerance, insulin resistance and reduced the area under the curve (AUC) in T2DM animals (Figures 1C-E).

\section{Exenatide Protects Against Cardiac Dysfunction in Diabetic Mice}

To assess whether exenatide protects against the development of diabetic cardiomyopathy in diabetic mice, echocardiography 
A

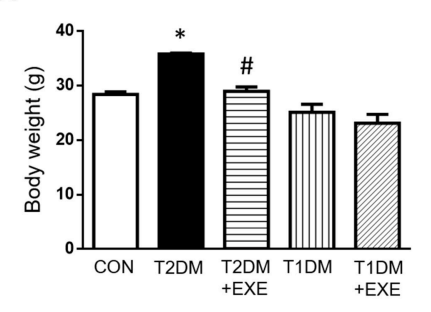

C

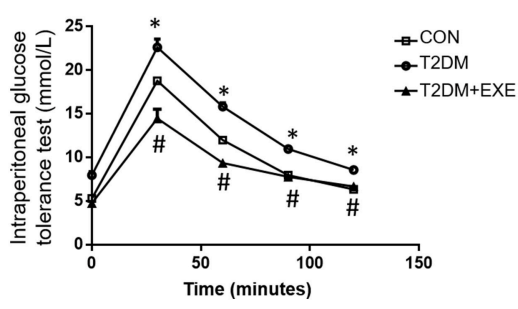

B

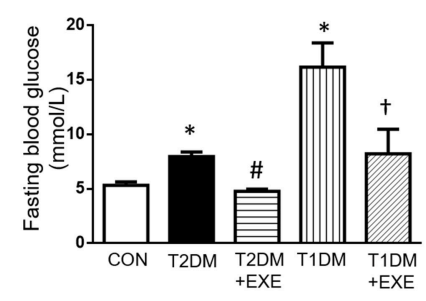

D

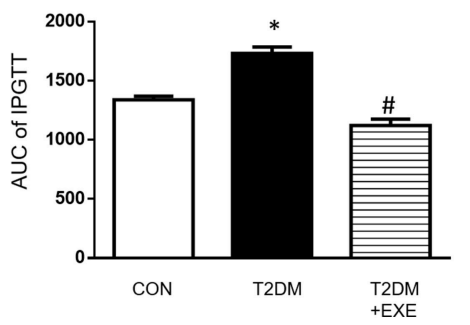

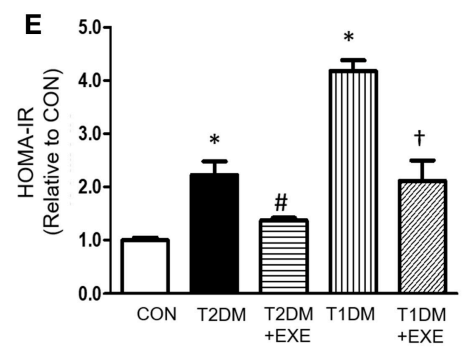

FIGURE 1 | Effects of exenatide on body weight and glucose tolerance in type 1 and type 2 diabetic mice. Type 1 and type 2 diabetic models were constructed as described in the section Materials and Methods. Either saline or exenatide ( $24 \mathrm{nmol} / \mathrm{kg} / \mathrm{d}$ ) was injected intraperitoneally daily for 4 weeks. Body weight (A), fasting blood glucose levels (B), intraperitoneal glucose tolerance test (IPGTT) results (C), area under curve for IPGTT (D) and HOMA-IR levels (E). Values are the mean \pm S.E.M. of 7-10 animals per group. ${ }^{*} p<0.05$ vs. control group, $\# p<0.05$ vs. T2DM group, ${ }^{\dagger} p<0.05$ vs. T1DM group.

was performed on CON, T2DM, T2DM+EXE and T1DM, T1DM+EXE animals one week prior to sacrifice. EF\%, LVSD, and LVDD were used to determine systolic function, and FS\% and E/A were used to determine diastolic function. Systolic and diastolic dysfunction developed in T2DM and T1DM animals in comparison to CON mice, and exenatide treatment of T2DM animals attenuated this dysfunction (Figure 2A). LVSD was increased in the T1DM group compared to CON group; however, there was no significant difference between the T2DM and CON groups (Figure 2E), and LVDD was decreased in the T2DM group compared to the CON group (Figure 2F). E/A, FS, and EF\% were all significantly decreased in T2DM and T1DM animals compared to CON mice. Treatment of T2DM animals with exenatide restored LVDD, E/A, FS, and EF\% to that of CON animals (Figures 2B-D,F). Moreover, treatment of T1DM animals with exenatide restored only E/A to that of CON animals (Figure 2D). Thus, exenatide treatment attenuates both systolic and diastolic dysfunction in type 2 diabetic mice and attenuates only diastolic dysfunction in T1DM mice.

\section{Exenatide Treatment Increases Cardiac MnSOD and Catalase and Reduces P53 in Diabetic Mouse Heart}

It was previously demonstrated that exenatide treatment attenuated myocardial infarction-induced cardiac injury through reduced ROS production (20). MnSOD and catalase are important enzymes that protect the cell from oxidative damage. We examined MnSOD and catalase levels in CON, T2DM, T2DM+EXE, T1DM, and T1DM+EXE animals. The levels of MnSOD and catalase protein were significantly reduced in T2DM and T1DM animals compared to CON mice.
Exenatide-treated T2DM and T1DM animals showed significant increases in MnSOD and catalase compared to model animals (Figures 2G-I).

P53 is known to initiate apoptosis upon DNA damage. We examined cardiac p53 levels in CON, T2DM, T2DM+EXE, T1DM, and T1DM+EXE animals. The level of p53 protein was significantly increased in T2DM and T1DM animals compared to CON mice. Exenatide-treated T2DM and T1DM animals exhibited significantly decreased p53 levels compared to diabetic animals (Figures 2G,J). Thus, exenatide treatment activates cardiac antioxidative activity and reduces P53 activity in diabetic cardiomyopathy mice.

\section{Exenatide Treatment Attenuates High Glucose-Induced ROS Production in H9c2 Cardiomyoblasts by Increasing Antioxidant Enzymes}

H9c2 myoblasts are a cell model used as an alternative for cardiomyocytes. It has been widely used for diabetes-related studies in vitro $(21,22)$. Therefore, we used this cell line to investigate the molecular mechanisms underlying the protective effects of exenatide.

$\mathrm{H} 9 \mathrm{c} 2$ cardiomyoblasts were incubated for $24 \mathrm{~h}$ with various concentrations of exenatide $(0-50 \mathrm{nM})$, and cytotoxicity was examined. Incubation of cells with $0-50 \mathrm{nM}$ exenatide was not cytotoxic (Figure 3A).

To further determine the effects of exenatide on oxidative stress induced by high glucose, the ROS levels were measured after exenatide treatment ( 25 or $50 \mathrm{nM}$ ) in the presence of high glucose $(35 \mathrm{mM})$ in $\mathrm{H} 9 \mathrm{c} 2$ cells. ROS levels were significantly increased by high-glucose stimulation compared with those in 


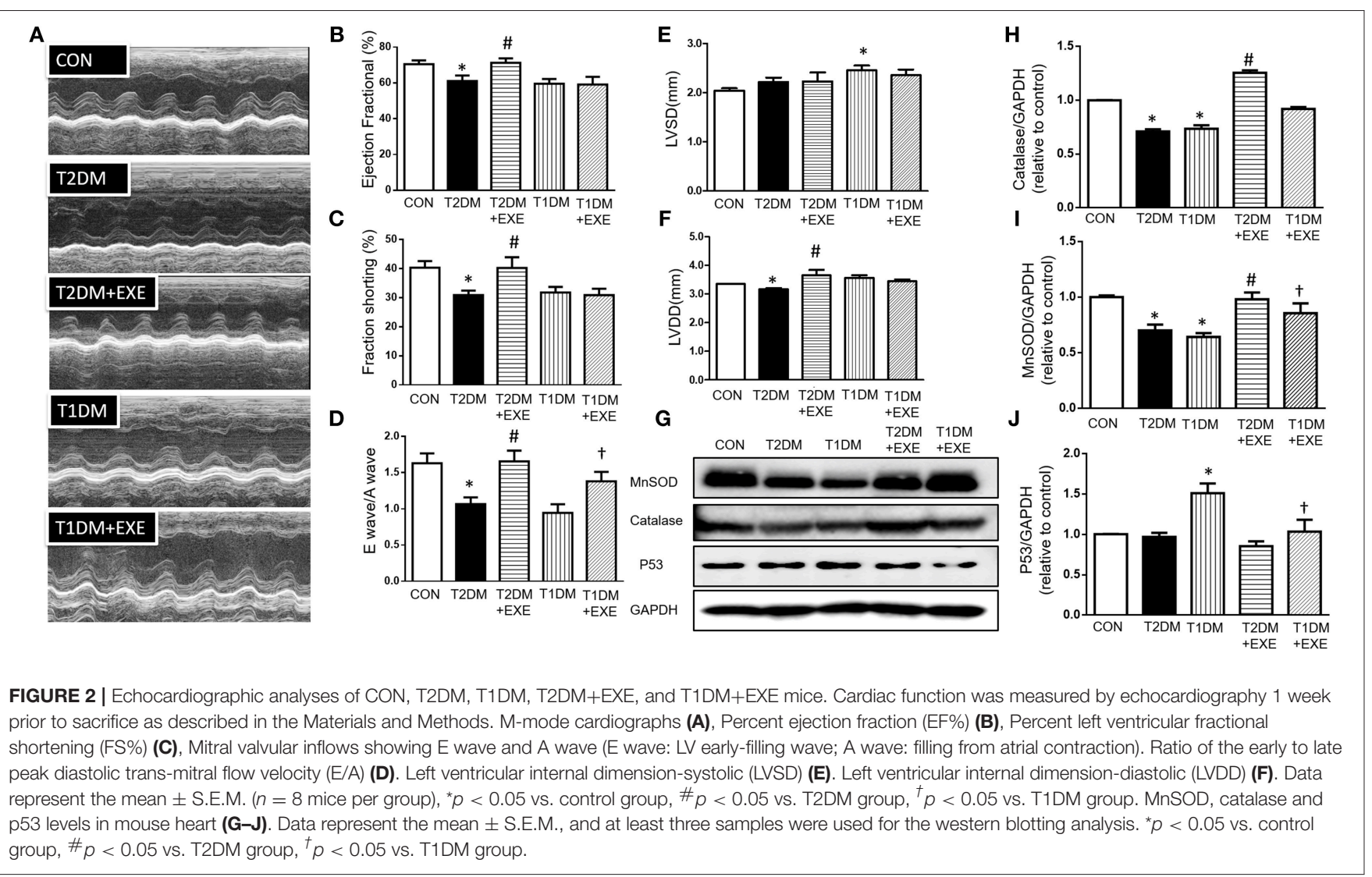

the control group $(P<0.05)$. Exenatide at concentrations of 25 and $50 \mathrm{nM}$ for $24 \mathrm{~h}$ decreased the ROS level (Figure 3B). The results were consistent with a qualified test of ROS, which was measured by the fluorescence intensity of cells by a fluorescence microplate reader at 488/525 $\mathrm{nm}$ (Figure 3C). Rosup was used as a positive control.

Then, levels of the antioxidant enzymes MnSOD and catalase were measured in $\mathrm{H} 9 \mathrm{c} 2$ cells after exenatide (25 or $50 \mathrm{nM}$ ) treatment in the presence of high glucose $(35 \mathrm{mM})$. MnSOD and catalase levels in the high-glucose group were significantly decreased compared with those in the control group $(P<$ $0.05)$. Exenatide treatment at concentrations of 25 and $50 \mathrm{nM}$ for $24 \mathrm{~h}$ increased the MnSOD $(P<0.05)$ and catalase levels $(P<0.05)$ (Figures 3D-F). These results indicated that exenatide reduced oxidative stress induced by high glucose by increasing the concentration of antioxidant defense enzymes in H9c2 cells.

\section{Exenatide Treatment Attenuates High Glucose-Induced Apoptosis in H9c2 Cells}

We next examined cell apoptosis in high glucose-treated cells. $\mathrm{H} 9 \mathrm{c} 2$ cells were incubated in the absence or presence of $35 \mathrm{mM}$ glucose in combination with exenatide or the catalase inhibitor 3AT or both. H9c2 cells incubated with $35 \mathrm{mM}$ glucose exhibited in a significant increase in cell apoptosis compared to the control cells (Figure 4A). Treatment of H9c2 cells with exenatide (25 or $50 \mathrm{nM}$ ) attenuated the observed apoptosis in cells induced by high glucose. Further, incubation of high glucose-treated cells with exenatide $(50 \mathrm{nM})$ in the presence of 3 -AT resulted in an elevation of cell apoptosis compared with exenatide treatment alone (Figures 4A,B). Thus, exenatide treatment attenuates high glucose-induced cell apoptosis in H9c2 cells.

Since high glucose-induced cell apoptosis results from an increase in ROS, we examined whether inhibiting antioxidant capacity could reverse this process. H9c2 cells were incubated in the absence or presence of $35 \mathrm{mM}$ glucose in combination with exenatide or the catalase inhibitor 3-AT or both. As shown in Figure 4C, incubation of $\mathrm{H} 9 \mathrm{c} 2$ cells with high glucose increased ROS production compared to the control treatment. Treatment of high glucose-incubated cells with exenatide decreased ROS compared to the control treatment (Figure 4C). Cells coincubated with high glucose and 3-AT displayed in a further increase in ROS levels than cells treated with high glucose alone. The presence of 3-AT attenuated the exenatide-induced reduction in ROS in high glucose-incubated cells (Figure 4C). The antioxidant protein expression levels were consistent with ROS production. Incubation of H9c2 cells with high glucose decreased MnSOD and catalase compared to the control condition (Figures $\mathbf{5 A}-\mathbf{C}$ ). The exenatide-induced elevation of catalase was attenuated by 3 -AT. The reduction in p53 by exenatide treatment was also diminished by 3AT (Figures 5A,B,D), indicating that exenatide exerts its antiapoptotic effects via elevating MnSOD and catalase levels, thus attenuating ROS production. 
A

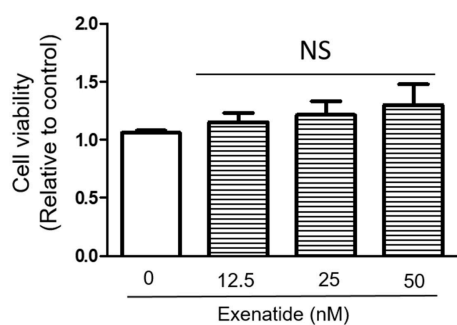

D

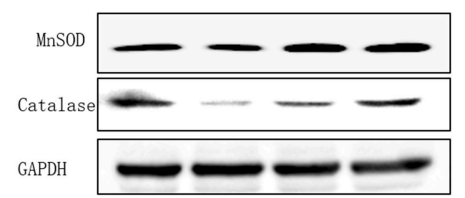

High glucose $(35 \mathrm{mM}) \quad-\quad+\quad+\quad+\quad+\quad+$

$+\quad+$

$\begin{array}{llllll}\text { Exenatide } 25 \mathrm{nM} & - & - & + & -\end{array}$
B

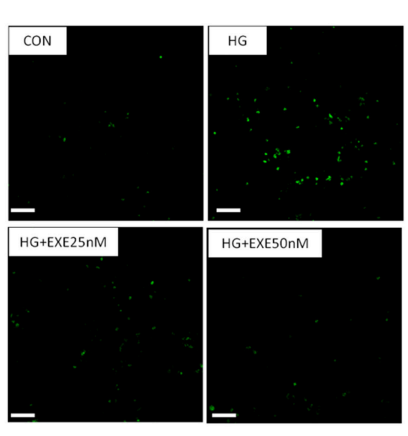

E

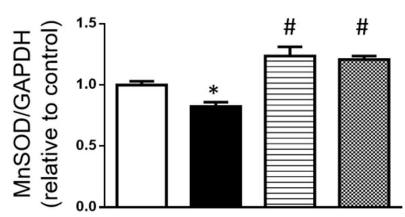

High glucose $(35 \mathrm{mM})-$

Exenatide $25 \mathrm{nM}$

Exenatide 50nM
C

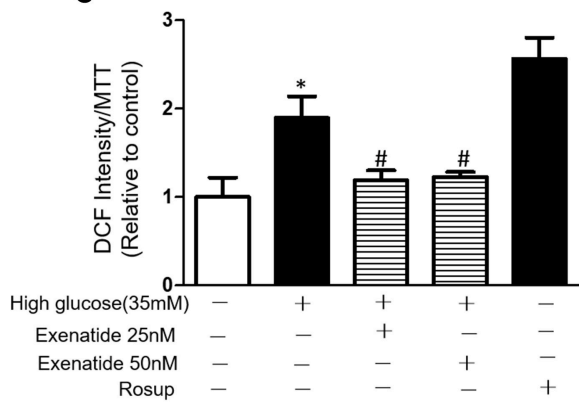

F

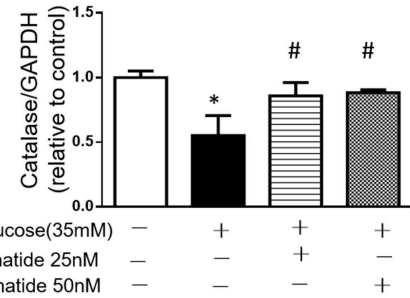

FIGURE 3 | Effects of exenatide on ROS production and antioxidant enzymes in high glucose-induced H9c2 cells. H9c2 cells were incubated with 0-50 nM concentrations of exenatide, and cell viability was determined as described in the Materials and Methods. Cell viability (A). H9c2 cells were incubated with 35 mM glucose in the presence or absence of exenatide ( 25 or $50 \mathrm{nM}$ ), and ROS production was determined as described in the Materials and Methods. Representative ROS fluorescence intensities are depicted; white scale bar, $50 \mu \mathrm{m}$ (B). A separate experiment for ROS production measured by the fluorescence intensity of cells using a fluorescence microplate reader at $488 / 525 \mathrm{~nm}$ (C). H9c2 cells were incubated with $35 \mathrm{mM}$ glucose in the presence or absence of exenatide (25 or $50 \mathrm{nM}$ ), and MnSOD, catalase and p53 levels were measured by western blotting as described in the Materials and Methods. A representative micrograph is depicted (D), MnSOD expression (E) catalase expression (F). Data represent the mean \pm S.E.M. The data represent at least three separate experiments, and each group has at least 3 samples. ${ }^{*} p<0.05$ vs. control group, $\# p<0.05$ vs. high-glucose group.
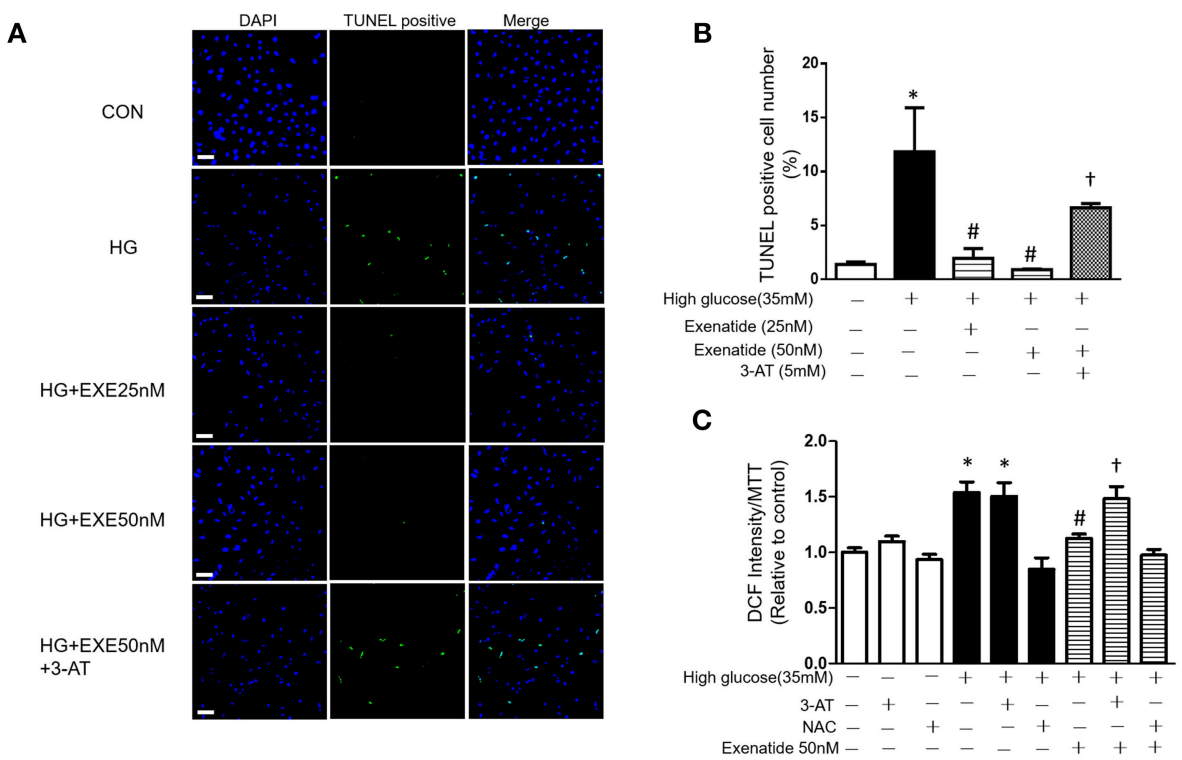

FIGURE 4 | Exenatide treatment attenuates high glucose-induced apoptosis in H9c2 cells. H9c2 cells were incubated in the absence or presence of high glucose in combination with exenatide or the catalase inhibitor 3-AT or both. Cell apoptosis was measured by TUNEL assay as described in the Materials and Methods: green, TUNEL-positive myocyte nuclei; blue, DAPI-stained nuclei. Representative TUNEL staining is depicted, white scale bar, $50 \mu \mathrm{m}$ (A), quantitative analysis of apoptosis (B). ROS production was measured by fluorescence intensity at 488/525 nm (C). Data are shown as the mean \pm S.E.M. The data represent at least three separate experiments, and each group has at least 3 samples. ${ }^{*} p<0.05$ vs. control group, ${ }^{\#} p<0.05$ vs. high-glucose group, ${ }^{\dagger} p<0.05$ vs. high-glucose plus exenatide group. 


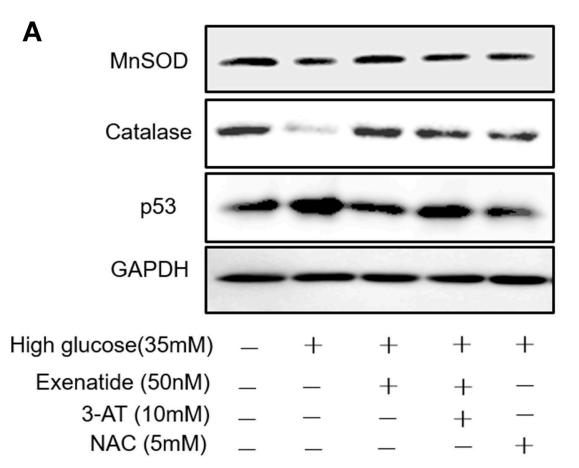

C

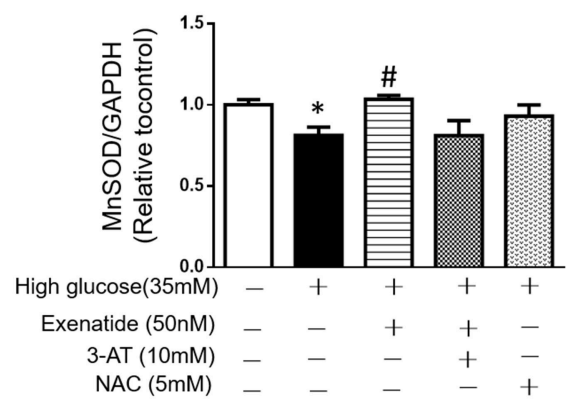

B

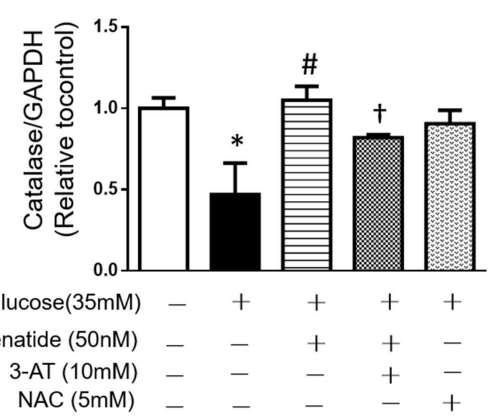

D

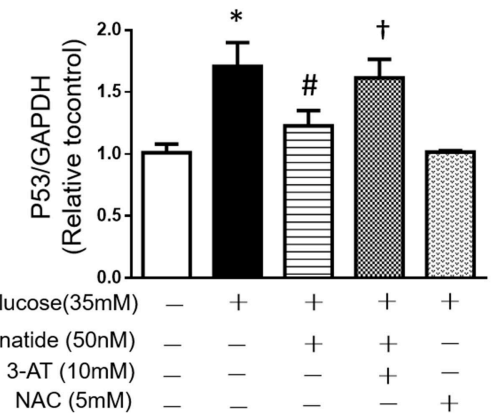

FIGURE 5 | Exenatide prevents cell apoptosis by increasing catalase in H9c2 cells incubated with high glucose. H9c2 cells were incubated in the absence or presence of high glucose in combination with exenatide or the catalase inhibitor 3-AT or both, and the expression of catalase, MnSOD and p53 was determined as described in the Materials and Methods. Representative western blots are depicted (A), catalase expression (B), MnSOD expression (C), and p53 expression (D). Data are shown as the mean \pm S.E.M. The data represent at least three separate experiments, and each group has at least 3 samples. ${ }^{*} p<0.05$ vs. control group, ${ }^{\#} p<0.05$ vs. high-glucose group. ${ }^{\dagger} p<0.05$ vs. high-glucose+Exenatide group.

\section{DISCUSSION}

In the present study, we found that exenatide protected cardiac function in both type 1 and type 2 diabetic mice. In addition, exenatide simultaneously attenuated ROS production through increased antioxidant enzyme levels, which were observed both in mouse hearts and $\mathrm{H} 9 \mathrm{c} 2$ cardiomyocytes. Moreover, inhibition of catalase by a catalase inhibitor (3-AT) diminished exenatide's protective effect on cell survival. These observations suggested that regulation of ROS production may be involved in the exenatide-mediated improvement of cardiac function in diabetic mouse hearts.

The T1DM and T2DM mouse models we used in the current study are two well-developed diabetic mouse models and have been used extensively in studies related to diabetes (23-25). T1DM is characterized by impaired beta cells and decreased insulin secretion. STZ was used as a method of $\beta$ cell ablation. This model exhibits extremely high glucose levels due to insufficient insulin secretion by islets $(23,26,27)$. Highfat diet-induced type 2 diabetes is characterized by obesity and insulin resistance. The high-fat diets used in this study contain a high content of saturated fatty acids $(45 \% \mathrm{kcal}$ of fat, mainly containing lard and soy oil). This kind of model is extensively used in studies of obesity, hepatic steatosis, type 2 diabetes and type 2 diabetes-related complications (28-30). This model exhibits normal or mildly elevated FBG levels with significant insulin resistance.

The doses of exenatide we chose in this study are based on previous studies. In humans, clinical administration of exenatide $(10 \mu \mathrm{g})$ twice daily or exenatide $(2 \mathrm{mg})$ once weekly as an addon therapy to type 2 diabetic patients was associated with greater improvements in several cardiovascular risk markers (7, 31-33). In animal studies, the doses of exenatide are different according to the aim of the therapeutic target. Exenatide $(500 \mu \mathrm{g} / \mathrm{kg})$ administered daily for 9 months promoted beneficial effects on short- and long-term memory performance in presenilin1 knock-in (PS1-KI) mice, a preclinical model of amyloidindependent neuronal dysfunction, and exenatide $(500 \mu \mathrm{g} / \mathrm{kg})$ was administered daily for 2 months to delay age-dependent cognitive decline in normal adult mice $(34,35)$. A molecular mechanism study of intestinal growth used exenatide $(42 \mu \mathrm{g} / \mathrm{kg}$, twice daily, equal to $10 \mathrm{nmol} / \mathrm{kg}$ ) as a GLP-1R agonist (36). In a T2DM mouse model induced by a high-fat diet, $20 \mu \mathrm{g} / \mathrm{kg} / \mathrm{d}$ exenatide (equal to $4.8 \mathrm{nmol} / \mathrm{kg} / \mathrm{d}$ ) or $100 \mu \mathrm{g} / \mathrm{kg} / \mathrm{d}$ exenatide (equal to $24 \mathrm{nmol} / \mathrm{kg} / \mathrm{d}$ ) was used to investigate its effects on the mechanisms of vascular aging in atherosclerosis or hepatic steatosis (37-40). Further, studies using exenatide ( $24 \mathrm{nmol} / \mathrm{kg} / \mathrm{d}$ ) to reverse the cardiac and cognitive dysfunction observed in high-fat diet-induced T2DM mice $(41,42)$. As our study used a similar model, we chose $24 \mathrm{nmol} / \mathrm{kg} / \mathrm{d}$ for the dose of exenatide as a therapeutic agent for both T1DM and T2DM mice. 
Exenatide was reported to protect cardiac function by antiinflammation or autophagy upregulation (43). Additionally, exenatide attenuates cardiac hypertrophy and prevents cardiac apoptosis via AMPK activation (44, 45). Therefore, exenatide's cardioprotective effects may be related to multiple signaling pathways. Oxidative stress and the accelerated ROS production induced by high glucose are known to play key roles in the progression of diabetic cardiovascular disease and cardiomyocyte apoptosis. High plasma glucose-induced oxidative stress, typified by ROS production, promotes increased damage to DNA, RNA, proteins, mitochondria and cell membranes, triggering a series of maladaptive stimuli that result in alterations in signal transduction and gene expression that can lead to cell death $(46,47)$. The key initial step in ROS formation is the conversion of molecular oxygen $\left(\mathrm{O}_{2}\right)$ to superoxide $\left(\mathrm{O}_{2^{-}}\right)$. Superoxide dismutases (MnSOD and $\mathrm{ZnSOD}$ ) are key enzymes that neutralize $\mathrm{O}_{2^{-}}$into less reactive hydrogen peroxide $\left(\mathrm{H}_{2} \mathrm{O}_{2}\right)$, which is then reduced to $\mathrm{H}_{2} \mathrm{O}$ by catalase or glutathione peroxidase. In this case, the intracellular antioxidative ability to increase ROS levels is largely determined by the expression of the enzymes SOD, catalase, and glutathione peroxidase (GSH-Px). It has been reported that exenatide treatment inhibits ROS production and attenuates fibrotic islet destruction in apoptotic pancreatic beta cells induced by fatty acids (48). In our study, we further found that in diabetic mouse heart and high glucosestimulated cardiac cells, exenatide attenuated ROS production by increasing catalase and MnSOD expression, suggesting that the induction of antioxidant enzymes may underlie exenatide's protective effects.

P53 is closely related to cardiac cell apoptosis. Activation of $\mathrm{p} 53$ reduces the expression of genes opposing cell death, such as $\mathrm{Bcl}-2$, and upregulates genes promoting apoptosis, such as Bax, therefore promoting cell apoptosis (49). In diabetic mice, suppressing $\mathrm{p} 53$ and the $\mathrm{Bax} / \mathrm{Bcl}-2$ ratio prevented progenitor cell apoptosis (50). P53 is also known to be induced by ROS (51). Antioxidant enzymes also affect p53 expression, and specific kidney overexpression of catalase reduces p53 levels and prevents apoptosis in mouse renal proximal tubules (52). In our present study, we found

\section{REFERENCES}

1. Gaede P, Oellgaard J, Carstensen B, Rossing P, Lund-Andersen H, Parving $\mathrm{HH}$, et al. Years of life gained by multifactorial intervention in patients with type 2 diabetes mellitus and microalbuminuria: 21 years followup on the Steno-2 randomised trial. Diabetologia. (2016) 59:2298-2307. doi: 10.1007/s00125-016-4065-6

2. Matheus AS, Tannus LR, Cobas RA, Palma CC, Negrato CA, GomesMB. Impact of diabetes on cardiovascular disease: an update. Int J Hypertens. (2013) 2013:653789. doi: 10.1155/2013/653789

3. Leon BM, Maddox TM. Diabetes and cardiovascular disease: Epidemiology, biological mechanisms, treatment recommendations and future research. World J Diabetes. (2015) 6:1246-58. doi: 10.4239/wjd.v6.i1 3.1246

4. Zheng X, Xu F, Liang H, Cao H, Cai M, Xu W, et al. SIRT1/HSF1/HSP pathway is essential for exenatide-alleviated, lipid-induced hepatic endoplasmic reticulum stress. Hepatology. (2017) 66:809-24. doi: 10.1002/hep.29238 increased expression of p53 in diabetic mouse hearts, and exenatide treatment reduced p53 levels and increased catalase and MnSOD levels, suggesting that the antiapoptotic effects of exenatide is mediated, at least in part, by a reduction in ROS.

In summary, our current findings demonstrate that exenatide treatment improves cardiac dysfunction in diabetic mice and attenuates apoptosis in high glucose-induced $\mathrm{H} 9 \mathrm{c} 2$ cardiomyoblasts. The mechanisms underlying these beneficial effects are a reduction in ROS production via increased activation of MnSOD and catalase and reduced P53 activation. We hypothesize that exenatide may be a candidate drug for the treatment of diabetic-related cardiovascular disease.

\section{ETHICS STATEMENT}

This study was carried out in accordance with the recommendations of the tips in the Guide for the Care and Use of Laboratory Animals of the Institutional Animal Care and Use Committee, China. The protocol was approved by the Ethics Committee for the Use of Experimental Animals of Qingdao University.

\section{AUTHOR CONTRIBUTIONS}

JW and XZ designed the study. WD, WC, and JW drafted and wrote the manuscript. WD, WC, DX, XG, YL, and DD performed the experiments and statistical analyses. All authors have read and approved the final version of the manuscript.

\section{FUNDING}

All sources of funding received for the research being submitted.

\section{ACKNOWLEDGMENTS}

This work was supported by grants from the Natural Science Foundation of ShanDong Province (JQ201815, ZR2017BH087) and the National Natural Science Foundation of China (81700704).
5. Xu F, Li Z, Zheng X, Liu H, Liang $\mathrm{H}$, Xu H, et al. SIRT1 mediates the effect of GLP-1 receptor agonist exenatide on ameliorating hepatic steatosis. Diabetes. (2014) 63:3637-46. doi: 10.2337/db14-0263

6. Scalzo RL, Moreau KL, Ozemek C, Herlache L, McMillin S, Gilligan S, et al. Exenatide improves diastolic function and attenuates arterial stiffness but does not alter exercise capacity in individuals with type 2 diabetes. J Diabetes Complicat. (2017) 31:449-55. doi: 10.1016/j.jdiacomp.2016.10.003

7. Simo R, Guerci B, Schernthaner G, Gallwitz B, Rosas-Guzman J, Dotta F, et al. Long-term changes in cardiovascular risk markers during administration of exenatide twice daily or glimepiride: results from the European exenatide study. Cardiovasc Diabetol. (2015) 14:116. doi: 10.1186/s12933-0150279-Z

8. Woo JS, Kim W, Ha SJ, Kim JB, Kim SJ, Kim WS, et al. Cardioprotective effects of exenatide in patients with ST-segment-elevation myocardial infarction undergoing primary percutaneous coronary intervention: results of exenatide myocardial protection in revascularization study. Arterioscler Thromb Vasc Biol. (2013) 33:2252-60. doi: 10.1161/ATVBAHA.113.301586 
9. Wang D, Luo P, Wang Y, Li W, Wang C, Sun D, et al. Glucagonlike peptide-1 protects against cardiac microvascular injury in diabetes via a cAMP/PKA/Rho-dependent mechanism. Diabetes. (2013) 62:1697-708. doi: $10.2337 / \mathrm{db} 12-1025$

10. Lee KH, Ha SJ, Woo JS, Lee GJ, Lee SR, Kim JW, et al. Exenatide prevents morphological and structural changes of mitochondria following ischaemia-reperfusion injury. Heart Lung Circ. (2017) 26:519-23. doi: 10.1016/j.hlc.2016.08.007

11. Lee KH, Cho H, Lee S, Woo JS, Cho BH, Kang JH, et al. Enhanced-autophagy by exenatide mitigates doxorubicin-induced cardiotoxicity. Int J Cardiol. (2017) 232:40-7. doi: 10.1016/j.ijcard.2017.01.123

12. Wang S, Ren J. Role of autophagy and regulatory mechanisms in alcoholic cardiomyopathy. Biochim Biophys Acta Mol Basis Dis. (2018) 1864:2003-9. doi: 10.1016/j.bbadis.2018.03.016

13. Leonardini A, D’Oria R, Incalza MA, Caccioppoli C, Andrulli Buccheri V, Cignarelli A, et al. GLP-1 receptor activation inhibits palmitate-induced apoptosis via ceramide in human cardiac progenitor cells. J Clin Endocrinol Metab. (2017) 102:4136-47. doi: 10.1210/jc.2017-00970

14. Chang G, Zhang D, Liu J, Zhang P, Ye L, Lu K, et al. Exenatide protects against hypoxia/reoxygenation-induced apoptosis by improving mitochondrial function in H9c2 cells. Exp Biol Med. (2014) 239:414-22. doi: $10.1177 / 1535370214522177$

15. Younce CW, Burmeister MA, Ayala JE. Exendin-4 attenuates high glucoseinduced cardiomyocyte apoptosis via inhibition of endoplasmic reticulum stress and activation of SERCA2a. Am J Physiol Cell Physiol. (2013) 304:C50818. doi: 10.1152/ajpcell.00248.2012

16. Faria A, Persaud SJ. Cardiac oxidative stress in diabetes: mechanisms and therapeutic potential. Pharmacol Ther. (2017) 172:50-62. doi: 10.1016/j.pharmthera.2016.11.013

17. Giacco F, Brownlee M. Oxidative stress and diabetic complications. Circ Res. (2010) 107:1058-70. doi: 10.1161/CIRCRESAHA.110.223545

18. Shah MS, Brownlee M. Molecular and cellular mechanisms of cardiovascular disorders in diabetes. Circ Res. (2016) 118:1808-29. doi: 10.1161/CIRCRESAHA.116.306923

19. Rhee KJ, Lee CG, Kim SW, Gim DH, Kim HC, Jung BD. Extract of ginkgo biloba ameliorates streptozotocin-induced type 1 diabetes mellitus and highfat diet-induced Type 2 diabetes mellitus in mice. Int J Med Sci. (2015) 12:987-94. doi: 10.7150/ijms.13339

20. Chang G, Zhang D, Yu H, Zhang P, Wang Y, Zheng A, et al. Cardioprotective effects of exenatide against oxidative stress-induced injury. Int J Mol Med. (2013) 32:1011-20. doi: 10.3892/ijmm.2013.1475

21. Gesmundo I, Miragoli M, Carullo P, Trovato L, Larcher V, Di Pasquale E, et al. Growth hormone-releasing hormone attenuates cardiac hypertrophy and improves heart function in pressure overload-induced heart failure. Proc Natl Acad Sci USA. (2017) 114:12033-8. doi: 10.1073/pnas.1712612114

22. Johnson R, Dludla P, Joubert E, February F, Mazibuko S, Ghoor S, et al. Aspalathin, a dihydrochalcone C-glucoside, protects $\mathrm{H} 9 \mathrm{c} 2$ cardiomyocytes against high glucose induced shifts in substrate preference and apoptosis. Mol Nutr Food Res. (2016) 60:922-34. doi: 10.1002/mnfr.201500656

23. Damond N, Thorel F, Moyers JS, Charron MJ, Vuguin PM, Powers AC, et al. Blockade of glucagon signaling prevents or reverses diabetes onset only if residual beta-cells persist. Elife. (2016) 5:e13828. doi: 10.7554/eLife.13828

24. Nonogaki K, Strack AM, Dallman MF, Tecott LH. Leptin-independent hyperphagia and type 2 diabetes in mice with a mutated serotonin 5-HT2C receptor gene. Nat Med. (1998) 4:1152-6. doi: 10.1038/2647

25. Hennige AM, Burks DJ, Ozcan U, Kulkarni RN, Ye J, Park S, et al. Upregulation of insulin receptor substrate-2 in pancreatic beta cells prevents diabetes. J Clin Invest. (2003) 112:1521-32. doi: 10.1172/JCI 18581

26. Ruan Q, Wang T, Kameswaran V, Wei Q, Johnson DS, Matschinsky F, et al. The microRNA-21-PDCD4 axis prevents type 1 diabetes by blocking pancreatic beta cell death. Proc Natl Acad Sci USA. (2011) 108:12030-5. doi: 10.1073/pnas.1101450108

27. Wang D, Sun H, Song G, Yang Y, Zou X, Han P, et al. Resveratrol improves muscle atrophy by modulating mitochondrial quality control in stz-induced diabetic mice. Mol Nutr Food Res. (2018) 62:e1700941. doi: $10.1002 / \mathrm{mnfr} .201700941$
28. Cui L, Liu M, Chang X, Sun K. The inhibiting effect of the Coptis chinensis polysaccharide on the type II diabetic mice. Biomed Pharmacother. (2016) 81:111-9. doi: 10.1016/j.biopha.2016.03.038

29. Janssens S, Heemskerk MM, van den Berg SA, van Riel NA, Nicolay K, Willems van Dijk K, et al. Effects of low-stearate palm oil and high-stearate lard high-fat diets on rat liver lipid metabolism and glucose tolerance. Nutr Metab. (2015) 12:57. doi: 10.1186/s12986-015-0053-y

30. Sutton GM, Trevaskis JL, Hulver MW, McMillan RP, Markward NJ, Babin MJ, et al. Diet-genotype interactions in the development of the obese, insulinresistant phenotype of C57BL/6J mice lacking melanocortin-3 or-4 receptors. Endocrinology. (2006) 147:2183-96. doi: 10.1210/en.2005-1209

31. Blonde L, Pencek R, MacConell L. Association among weight change, glycemic control, and markers of cardiovascular risk with exenatide once weekly: a pooled analysis of patients with type 2 diabetes. Cardiovasc Diabetol. (2015) 14:12. doi: 10.1186/s12933-014-0171-2

32. Mentz RJ, Bethel MA, Gustavson S, Thompson VP, Pagidipati NJ, Buse JB, et al. Baseline characteristics of patients enrolled in the Exenatide Study of Cardiovascular Event Lowering (EXSCEL). Am Heart J. (2017) 187:1-9. doi: 10.1016/j.ahj.2017.02.005

33. Russell-Jones D, Cuddihy RM, Hanefeld M, Kumar A, Gonzalez JG, Chan $M$, et al. Efficacy and safety of exenatide once weekly versus metformin, pioglitazone, and sitagliptin used as monotherapy in drug-naive patients with type 2 diabetes (DURATION-4): a 26-week double-blind study. Diabetes Care. (2012) 35:252-8. doi: 10.2337/dc11-1107

34. Bomba M, Ciavardelli D, Silvestri E, Canzoniero LM, Lattanzio R, Chiappini P, et al. Exenatide promotes cognitive enhancement and positive brain metabolic changes in PS1-KI mice but has no effects in 3xTg-AD animals. Cell Death Dis. (2013) 4:e612. doi: 10.1038/cddis.2013.139

35. Bomba M, Granzotto A, Castelli V, Massetti N, Silvestri EL, Canzoniero MT, et al. Exenatide exerts cognitive effects by modulating the BDNFTrkB neurotrophic axis in adult mice. Neurobiol Aging. (2018) 64:33-43. doi: 10.1016/j.neurobiolaging.2017.12.009

36. Koehler JA, Baggio LL, Yusta B, Longuet C, Rowland KJ, Cao X, et al. GLP-1R agonists promote normal and neoplastic intestinal growth through mechanisms requiring Fgf7. Cell Metab. (2015) 21:379-91. doi: 10.1016/j.cmet.2015.02.005

37. Ghadieh HE, Muturi HT, Najjar SM. Exenatide prevents diet-induced hepatocellular injury in A CEACAM1-dependent mechanism. J Diabetes Treat. (2017) 2017. doi: 10.29011/2574.7568.000033

38. Shao N, Yu XY, Ma XF, Lin WJ, Hao M, Kuang HY. Exenatide delays the progression of nonalcoholic fatty liver disease in $\mathrm{C} 57 \mathrm{BL} / 6$ mice, which may involve inhibition of the NLRP3 inflammasome through the mitophagy pathway. Gastroenterol Res Pract. (2018) 2018:1864307. doi: 10.1155/2018/1864307

39. Wang Z, Hou L, Huang L, Guo J, Zhou X. Exenatide improves liver mitochondrial dysfunction and insulin resistance by reducing oxidative stress in high fat diet-induced obese mice. Biochem Biophys Res Commun. (2017) 486:116-23. doi: 10.1016/j.bbrc.2017.03.010

40. Yang $\mathrm{G}$, Lei $\mathrm{Y}$, Inoue $\mathrm{A}$, Piao $\mathrm{L}$, $\mathrm{Hu} \mathrm{L}$, Jiang $\mathrm{H}$, et al. Exenatide mitigated diet-induced vascular aging and atherosclerotic plaque growth in ApoE-deficient mice under chronic stress. Atherosclerosis. (2017) 264:1-10. doi: 10.1016/j.atherosclerosis.2017.07.014

41. Monji A, Mitsui T, Bando YK, Aoyama M, Shigeta T, Murohara T. Glucagon-like peptide-1 receptor activation reverses cardiac remodeling via normalizing cardiac steatosis and oxidative stress in type 2 diabetes. Am J Physiol Heart Circ Physiol. (2013) 305:H295-304. doi: 10.1152/ajpheart.009 90.2012

42. Gault VA, Porter WD, Flatt PR, Holscher C. Actions of exendin-4 therapy on cognitive function and hippocampal synaptic plasticity in mice fed a high-fat diet. Int J Obes. (2010) 34:1341-4. doi: 10.1038/ijo.2010.59

43. Tate M, Robinson E, Green BD, McDermott BJ, Grieve DJ. Exendin-4 attenuates adverse cardiac remodelling in streptozocin-induced diabetes via specific actions on infiltrating macrophages. Basic Res Cardiol. (2016) 111:1. doi: 10.1007/s00395-015-0518-1

44. Zhou Y, He X, Chen Y, Huang Y, Wu L, He J. Exendin-4 attenuates cardiac hypertrophy via AMPK/mTOR signaling pathway activation. Biochem Biophys Res Commun. (2015) 468:394-9. doi: 10.1016/j.bbrc.2015.09.179 
45. XiaoTian L, QiNan W, XiaGuang G, WuQuan D, Bing C, ZiWen L. Exenatide activates the APPL1-AMPK-PPARalpha axis to prevent diabetic cardiomyocyte apoptosis. J Diabetes Res. (2016) 2016:4219735. doi: 10.1155/2016/4219735

46. Lin N, Li XY, Zhang HM, Yang Z, Su Q. microRNA-199a-5p mediates high glucose-induced reactive oxygen species production and apoptosis in INS1 pancreatic beta-cells by targeting SIRT1. Eur Rev Med Pharmacol Sci. (2017) 21:1091-8.

47. Liang W, Chen M, Zheng D, Li J, Song M, Zhang W, et al. The opening of ATPsensitive $\mathrm{K}+$ channels protects $\mathrm{H} 9 \mathrm{c} 2$ cardiac cells against the high glucoseinduced injury and inflammation by inhibiting the ROS-TLR4-necroptosis pathway. Cell Physiol Biochem. (2017) 41:1020-34. doi: 10.1159/000461391

48. Kim JW, Park SY, You YH, Ham DS, Lee SH, Yang HK, ET AL. Suppression of ROS production by exendin-4 in PSC attenuates the high glucose-induced islet fibrosis. PLoS ONE. (2016) 11:e0163187. doi: 10.1371/journal.pone.0163187

49. Miyashita T, Reed JC. Tumor suppressor p53 is a direct transcriptional activator of the human bax gene. Cell. (1995) 80:293-9. doi: 10.1016/0092-8674(95)90412-3

50. Li H, Liu J, Wang Y, Fu Z, Huttemann M, Monks TJ, et al. MiR-27b augments bone marrow progenitor cell survival via suppressing the mitochondrial apoptotic pathway in Type 2 diabetes. Am J Physiol Endocrinol Metab. (2017) 313:E391-401. doi: 10.1152/ajpendo.00073.2017

51. Wen J, Shu Y, Zhang W. ROS, P53, and ischemic acute kidney injury in diabetic models. Kidney Int. (2015) 88:198-9. doi: 10.1038/ki. 2015.130

52. Brezniceanu ML, Liu F, Wei CC, Tran S, Sachetelli S, Zhang SL, et al. Catalase overexpression attenuates angiotensinogen expression and apoptosis in diabetic mice. Kidney Int. (2007) 71:912-23. doi: 10.1038/sj.ki.5 002188

Conflict of Interest Statement: The authors declare that the research was conducted in the absence of any commercial or financial relationships that could be construed as a potential conflict of interest.

Copyright (C) 2019 Ding, Chang, Guo, Liu, Xiao, Ding, Wang and Zhang. This is an open-access article distributed under the terms of the Creative Commons Attribution License (CC BY). The use, distribution or reproduction in other forums is permitted, provided the original author(s) and the copyright owner(s) are credited and that the original publication in this journal is cited, in accordance with accepted academic practice. No use, distribution or reproduction is permitted which does not comply with these terms. 